Textures and Microstructures, Vol. 33, pp. 321-328 Reprints available directly from the publisher Photocopying permitted by license only
(C) 1999 OPA (Overseas Publishers Association) N.V.

Published by license under the Gordon and Breach Science Publishers imprint. Printed in Malaysia.

\title{
ACOUSTIC RESIDUAL STRESS ANALYSIS IN TEXTURED ALUMINIUM ALLOYS
}

\author{
V.N. SEREBRYANY \\ All-Russia Institute of Light Alloys, 121596, Moscow, Russia
}

In biaxial stress state approach distributions of the residual stress in two types of aluminium textured semiproducts with axial and rhombic sample symmetry have been evaluated from the difference in velocities of orthogonally polarized ultrasonic shear bulk waves with account for a texture-dependent component of the total relative velocity change.

Keywords: Ultrasonics; Residual stress; Texture; Aluminium semiproducts

\section{INTRODUCTION}

The velocity of an ultrasonic wave passing through a polycrystalline material depends both on its state of stress and its texture (Sayers, 1982). This fact presents an opportunity for a nondestructive determination of both stress and texture by ultrasonic measurements. The main problem is to find away of separating the contributions of the stress and texture to the change in wave propagation velocity.

For biaxial stress state approach the experimental information about the principal stresses $\left(\sigma_{1}\right.$ and $\left.\sigma_{2}\right)$ of textured material were obtained from Eq. (1) when material symmetry directions coincide with principal stress axes (Clark and Moulder, 1985):

$$
A=\frac{2\left(V_{1}-V_{2}\right)}{V_{1}+V_{2}}=\left[\frac{2\left(V_{1}-V_{2}\right)}{V_{1}+V_{2}}\right]_{\text {texture }}+a\left(\sigma_{1}-\sigma_{2}\right) .
$$

Here, $A$ is an acoustic anisotropy parameter; $V_{1}$ and $V_{2}$ are velocities of the shear waves polarized along the $\sigma_{1}$ and $\sigma_{2}$ directions, respectively and $a$ is an acoustoelasic constant. 
In this paper we propose two acoustic techniques based on a solution of Eq. (1) for the determination of the residual stress in two types of the aluminium textured (cast and rolled) semiproducts with axial and rhombic sample symmetry.

\section{MATERIALS AND EXPERIMENTAL PROCEDURES}

This investigation was carried out on two types of aluminium semiproducts: (1) on templets cut from a cylindrical ingot of the aluminium alloy of grade D16 with diameter $260 \mathrm{~mm}$ transversely in axial direction of the ingot; and (2) on the templets cut from a thick hot rolled and quenched plate of width $430 \mathrm{~mm}$ of the aluminium alloy of grade V95 along transverse direction. The thicknesses of the first and second templets are equal to 60 and $15 \mathrm{~mm}$, respectively, so that the stresses aligned through these thicknesses are negligibly small. In consequence of this we received a biaxial stress state with the main stresses directed along radial and hoop directions for the first templet and directed along transverse and normal directions for the second templet. The basis for a given ultrasonic residual stress investigation is the acoustic anisotropy parameter measurement (Clark and Moulder, 1985). This parameter depends on the relation between the difference and sum of the velocities of the orthogonally polarized shear bulk waves. In this case the ultrasonic examination does not require measuring the density and thickness of samples investigated, and the phase velocity of the shear waves can be judged by time delays, i.e., the time of the ultrasonic wave propagation between the primary and the $n$-th reflected signal. Time delays were measured in an echo-pulse mode using acoustic piezoelectric transducers with a resonance frequency of $7-10 \mathrm{MHz}$ (Serebryany, 1994). These transducers send two shear ultrasonic waves: (1) along the axial direction of the cylindrical templet with the polarization planes of the waves oriented along and across the radial direction; (2) along the rolling direction of the plate templet with the polarization planes of the waves oriented along the transverse and normal directions, respectively. The time delay measurements are carried out in different points along the radial direction for the cylindrical templet and across thickness of the plate templet. For an evaluation of the absolute values of the residual stresses are defined acoustoelastic coefficients $(a)$ of the investigated samples using the mechanical tests (Serebryany and Kalmykov, 1996). 
For the ingots of grade D16 and the quenched plate of grade V95 the values of these coefficients are equal $-6.0 \cdot 10^{-5}$ and $-3.5 \cdot 10^{-5}$ $\mathrm{MPa}^{-1}$, respectively. The instrumental error of the time delay measurements does not exceed $8 \mathrm{~ns}$ for a $40 \mu$ s travel time for the ingot templet and a $20 \mu$ s travel time for the plate one. The relative errors of these measurements are equal to $2 \cdot 10^{-4}$ and $4 \cdot 10^{-4}$, respectively, they are equivalent to a stress uncertainty of about 8-10 MPa. The strain gauge stress analysis of the ingot templet was performed with using the "holetechnique" (Birger, 1963). The photoelastic stress analysis of the plate templet was performed in accordance with the known technique (Prigorovski, 1983).

\section{STRESS EVALUATION IN AXIAL SYMMETRY SAMPLES}

According to Eq. (1) in biaxial stress state approach the difference between the main stresses are defined from the ultrasonic measurements of the acoustic anisotropy parameter, if a texture depending term of this parameter is known. (Sayers, 1982) showed, that for cubic metals the texture depending term of the Eq. (1) is the directly proportional to the ODF coefficient $C_{4}^{20}$. In the axial symmetry semiproducts is formed an axial type of texture as the result of the different working operations (casting, extrusion and so on). The ODF coefficient $C_{4}^{20}$ is equal to zero for the axial texture type, and as a consequence the texture depending term of the acoustic anisotropy parameter is also equal to zero. For this case the stress-velocity relation (1) is given by

$$
\frac{2\left(V_{1}-V_{2}\right)}{V_{1}+V_{2}}=\frac{2\left(\tau_{2}-\tau_{1}\right)}{\tau_{1}+\tau_{2}}=a\left(\sigma_{1}-\sigma_{2}\right)
$$

where $\tau_{1}, \tau_{2}$ are time of passage through the templet thickness of two shear waves the polarization planes of which were oriented across and along the radial direction, respectively; $\sigma_{1}, \sigma_{2}$ are hoop and radial stresses. Then, we solve Eq. (2) for the difference $\left(\sigma_{1}-\sigma_{2}\right)$ of the principal stresses, measuring $\tau_{1}$ and $\tau_{2}$ values in different equally spaced points along the radial direction of the ingot templet. The ultrasonic $\left(\sigma_{1}-\sigma_{2}\right)$ values as a function of $r(r-\mathbf{a}$ current value of radius) are presented in Fig. 1. The ultrasonic values of the principal stresses are compared with similar ones, measured by strain gauges. The results of strain 


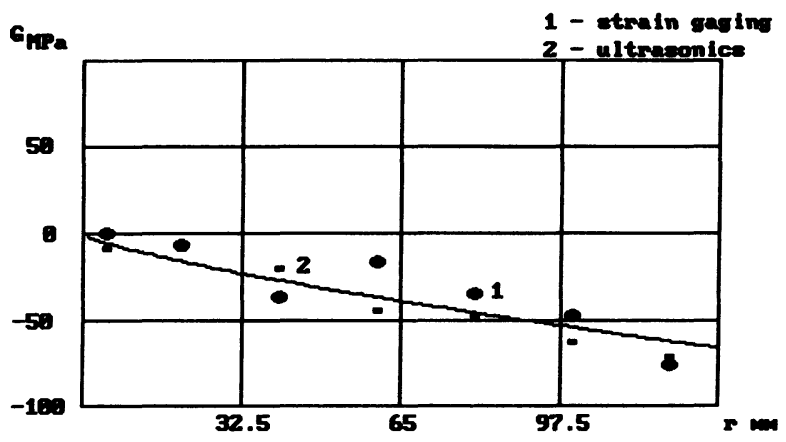

FIGURE $1 G(r)=\sigma_{1}(r)-\sigma_{2}(r)$-values defined from ultrasonic (2) and strain gauge measurement (1) data for the aluminium disk.

gauge measurements also are presented in Fig. 1. The results presented in Fig. 1 show that the ultrasonic $\left(\sigma_{1}-\sigma_{2}\right)$ values agree rather well within the limits of experimental errors with the strain gauge $\left(\sigma_{1}-\sigma_{2}\right)$ values.

In biaxial stress state approach for the given templet the distributions of residual stresses satisfy a stress equilibrium equation (Clark and Moudler, 1985)

$$
\frac{\mathrm{d} \sigma_{2}}{\mathrm{~d} r}=\frac{\sigma_{1}-\sigma_{2}}{r} .
$$

Then, we integrate Eq. (3) to obtain

$$
\sigma_{2}(r)=\sigma_{2}(R)+\int_{R}^{r} \frac{\sigma_{1}-\sigma_{2}}{r} \mathrm{~d} r
$$

where $R$ - a radius of the ingot templet.

At $r=R$ we have $\sigma_{2}=0$ (traction-free boundary). We fit the ultrasonic $\left(\sigma_{1}-\sigma_{2}\right)$ values as a function of $r$ to a polynomial of the form $\alpha(R / r)^{m}$, where $\alpha$ and $m$ - parameters, defined by a least-squares method. Then, we integrate (4), substituting $\left(\sigma_{1}-\sigma_{2}\right)$ for $\alpha(R / r)^{m}$ to obtain

$$
\sigma_{2}(r)=\frac{a}{m}\left[1-\left(\frac{R}{r}\right)^{m}\right]
$$

Finally, we use this form of $\sigma_{2}(r)$ in the relation

$$
\sigma_{1}(r)=\left(\sigma_{1}-\sigma_{2}\right)(r)+\sigma_{2}(r)
$$


to obtain $\sigma_{1}(r)$

$$
\sigma_{1}(r)=\frac{a}{m}\left[1+(m-1)\left(\frac{R}{r}\right)^{m}\right]
$$

The offered acoustic technique was applied for residual stress evaluation in the above-mentioned disks cut from the cylindrical ingot of the aluminium alloy of grade D16. The calculated and strain gauge values $\sigma_{2}(r)$ and $\sigma_{1}(r)$ of the ingot disk are presented in Figs. 2 and 3, respectively. The data presented in Figs. 2 and 3 show, that coincidence of the results on both methods is within the inaccuracy of the experiment.

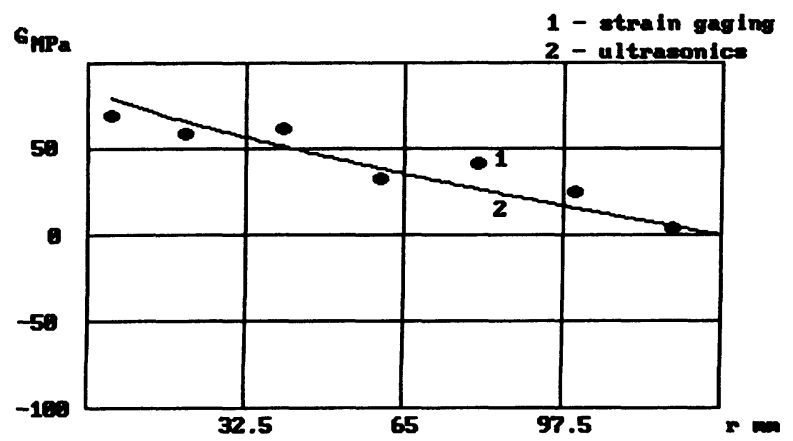

FIGURE 2 The radial stress values as a function of the current radial value defined from ultrasonic (2) and strain gauge measurement (1) data for the aluminium disk.

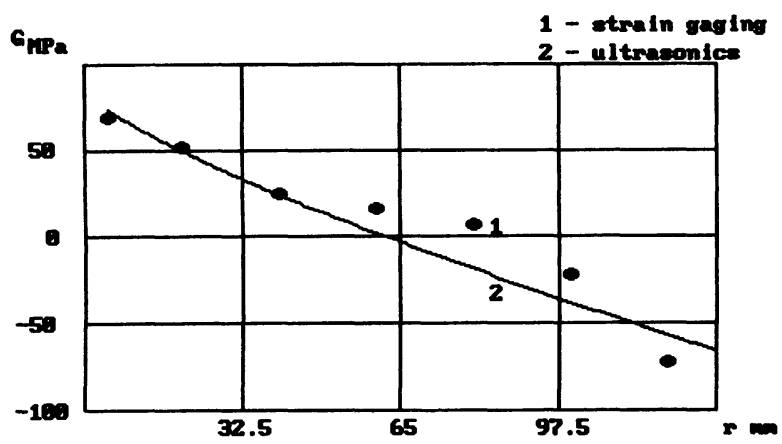

FIGURE 3 The hoop stress values as a function of the current radial value defined from ultrasonic (2) and strain gauge measurement (1) data for the aluminium disk. 


\section{STRESS EVALUATION IN RHOMBIC SYMMETRY SAMPLES}

A rolling type of texture is formed in the rhombic symmetry semiproducts (rolling plates, for example). In this case the ODF coefficient $C_{4}^{20}$ and in consequence the texture-depending term of the acoustic anisotropy parameter are not equal to zero (Sayers, 1982), as a consequence there is need to devise a method of separating the contributions of the stress and texture to the change in wave propagation velocity. For stress evaluation in rhombic symmetry samples we propose to use a stress-free reference sample cut from the investigated plate. Based on the above-mentioned time delay measurements carried out in the same points across thickness of the plate templet and stress-free sample the distribution of the $\left(\sigma_{1}-\sigma_{2}\right)$ stress difference across thickness of the quenched aluminium plate (where $\sigma_{1}$ and $\sigma_{2}$ are the transverse and normal stresses) can be obtained.

In the central part of the plate templet the normal stresses are negligible and we have an axial stress state along the transverse direction. On the contrary, because of the boundary conditions on the lateral edge of the plate templet the transverse stresses are equal to zero and we have the axial stress state along the normal direction. Then, for these two cases we can receive from Eq. (1) the distributions of the transverse and normal stresses across the thickness of the quenched aluminium plate. The results of these ultrasonic experiments are presented in Figs. 4 and 5, accordingly. These stress distributions are compared with similar

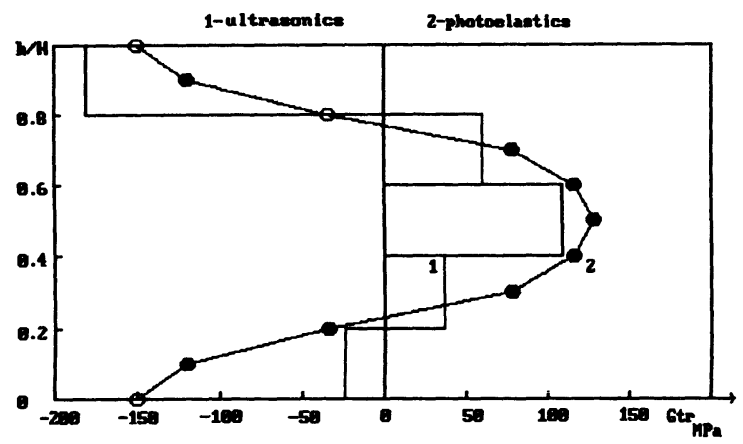

FIGURE 4 The transverse residual stress distribution through thickness of the central part of the plate templet. 


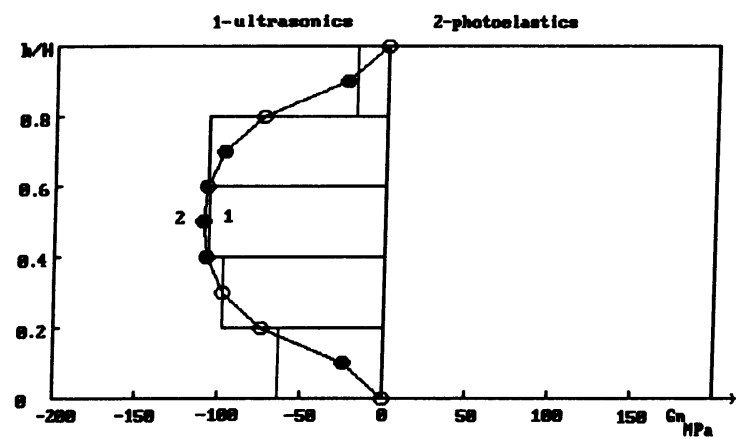

FIGURE 5 The normal residual stress distribution through thickness on the lateral edge of the plate templet.

distributions obtained by a photoelastic stress analysis. The photoelastic stress distributions also are presented in Figs. 4 and 5. The acoustic distributions of stresses are in quite a good conformity with the photoelastic ones.

\section{CONCLUSIONS}

The two acoustic techniques and the experimental results obtained with its employment can be used for the residual stress evaluation in axial and biaxial stress state in textured semiproducts by ultrasonic measurement of time delays of the volume shear waves. For the aluminium semiproducts of two types a good agreement of the ultrasonic results on the one hand and strain gauge and photoelastic results on the other hand was obtained. The proposed ways of separating the contributions of stress and texture to the change in shear wave propagation velocity also can be useful for texture and plastic anisotropy evaluation of stressed semiproducts by ultrasonic methods (Serebryany, 1996).

\section{Acknowledgments}

The author expresses his thanks to Mr. E. Kalmykov and.Dr. L. Byakov (All-Russia Institute of Light Alloys) for help in carrying out ultrasonic, strain gauge and photoelastic measurements. 


\section{References}

Birger, I.A. (1963). Residual Stresses (in Russian), Mashgiz, Moscow.

Clark, A.V., Jr. and Moulder, J.C. (1985). Residual stress determination in aluminium using electromagnetic acoustic transducers, Ultrasonics, 23(11), 253-259.

Prigorovski, N.L. (1983). Methods and Means of Strain and Stress Fields Determination (in Russian), M., Moscow.

Sayers, C.M. (1982). Ultrasonic velocities in anisotropic polycrystalline aggregates, J. Phys. D.: Appl. Phys., 15, 2157-2167.

Serebryany, V.N. (1994). Quantitative estimation of steel sheet texture by method of ultrasonic bulk waves, Ind. Lab., 60(4), 223-227.

Serebryany, V.N. and Kalmykov, E.B. (1996). Acoustic Anisotropy Parameter Application in Axial Products (in Russian). Abstracts of the 4th Int. Workshop Engineers and physical problems of new technology, held in Moscow, May 21-23, 1996, pp. 20-21, MSTU, Moscow.

Serebryany, V.N. (1996). Plastic anisotropy prediction by ultrasonic texture data, Textures and Microstructures, 25, 223-228. 